\title{
Towards understanding broad degeneracy in non-strange mesons
}

\author{
S.S. Afonin \\ October 5, 2018 \\ V.A. Fock Institute of Physics, St. Petersburg State University, \\ St. Petersburg 198504, ul. Ulyanovskaya 1, Russia \\ E-mail: afonin24@mail.ru
}

\begin{abstract}
The spectroscopic regularities of modern empirical data on the non-strange mesons up to $2.4 \mathrm{GeV}$ can be summarized as a systematic clustering of states near certain values of energy. It is getting evident that some unknown X-symmetry triggers the phenomenon. We review the experimental status of this symmetry and recent theoretical attempts put forward for explanation of broad degeneracy.
\end{abstract}

PACS: 12.38.Aw, 12.38.Qk, 14.40.-n

Keywords: Experimental spectrum; Hadron symmetries 


\section{Introduction}

The spectroscopic studies of light hadrons have a long history reflected in the numerous literature. In recent years many new data has appeared in the section "Further States" of Particle Data [1]. The emerging spectroscopic picture is quite intriguing and theoretical explanations are called for. This raised a renewed interest to the subject.

As was realized recently [2-6] the experimental spectrum of light nonstrange mesons reveals a broader degeneracy than one could expect from the approximate symmetries of QCD Lagrangian. For instance, the experimental spectrum points out an intriguing pattern of degeneracy among the meson states with different spin, see Fig. 1 below. It is getting evident that some symmetry of unknown nature triggers the phenomenon, we will refer to it as $\mathrm{X}$-symmetry.

It is quite usual in physics that all spectral symmetries of compound systems are not directly seen on the fundamental level. In the same way, it may be that the X-symmetry seen in Fig. 1 hardly can be envisaged directly from QCD, rather one should look into how the states are "constructed".

A popular assumption about the structure of mesons is the hypothesis that the mesons represent a gluon string with a quark/antiquark at the ends. We will review the typical predictions of the hadron string models and the linearly rising potentials and compare them with the available experimental data, of our concern will be the spectroscopic aspect of the problem.

Typically the hadron strings and linearly rising potentials predict the following law for the spectrum of excitations,

$$
M^{2} \sim a(L+b n)
$$

where $L$ is the angular momentum, $n$ is the radial quantum number, $a$ and $b$ are constants characterizing the angular and radial slopes, respectively. As the quark spin is $\frac{1}{2}$, the quark-antiquark pair can be either in the singlet $(s=0)$ or in the triplet $(s=1)$ states. The angular momentum $L$ is related to the total spin $J$ as

$$
\begin{array}{ll}
s=0: & J=L, \\
s=1: & J=L, L \pm 1 \quad(\text { for } L=0: J=L+1) .
\end{array}
$$

Recently it was proposed [5] that in reality $b=1$. We will scrutinize this proposal using the latest experimental data. As a byproduct we will obtain a form of the string-like spectrum which seems to be the most consistent with the available phenomenology. This analysis could be useful for future hadron string approaches, AdS/QCD methods, and other QCD-inspired models. 
The review is organized as follows. In Sect. 2 we consider some general aspects of quasiclassical string description for the light mesons. A phenomenological analysis of outlined picture is performed in Sect 3. The Sect. 4 is devoted to discussions of obtained results and of some related problems. We conclude in Sect. 5 .

\section{Quasiclassical hadron string picture}

Let us present some heuristic ideas leading to spectrum (1). For high radial or orbital excitation a meson state can be considered quasiclassically as a pair of relativistic quarks interacting via a confining linear potential. Consequently, neglecting the quark spin, the meson mass can be written as

$$
M=2 p+\sigma r
$$

where $p$ is the relativistic quark momentum and $\sigma$ is the mass density per unit length. The maximal length of chromoelectric flux tube between the quarks is

$$
l=\frac{M}{\sigma} .
$$

Applying the WKB method (see, e.g., [7]) one obtains the following quantization condition,

$$
\int_{0}^{l} p d r=\pi(n+\gamma), \quad n=0,1,2, \ldots
$$

Substituting the momentum $p$ from Eq. (3) one gets

$$
M^{2}=4 \pi \sigma(n+\gamma) .
$$

Here $\gamma$ is a constant of order of unity characterizing the nature of turning points (in a close vicinity of these points the semiclassical methods are not applicable). The correction $\gamma$ is known to be of importance for practical applications because it usually extends the applicability of the Bohr-Sommerfeld quantization (5) from $n \sim 10$ to $n \sim 1$. In some cases (e.g., the harmonic oscillator) the WKB approximation even provides the exact spectrum. The analysis performed in [8] showed that the accuracy of the WKB method in the given situation is at the level of several percents. As long as one deals with a centrosymmetrical potential in Eq. (3), the WKB method gives

$$
\gamma=\frac{1}{2}
$$


This value is quite remarkable: The same value $\frac{1}{2}$ is predicted by the LovelaceShapiro dual amplitude $[9,10]$ and in some channels it appeared naturally within the QCD sum rules [11-14]. For the S-wave states $(L=0)$, however, the situation is different and the WKB method yields

$$
\gamma_{s}=\frac{3}{4}
$$

On the other hand, the spectrum of rotating relativistic string is known to be linear in the angular momentum $L$ (Chew-Frautschi formula),

$$
M^{2}=2 \pi \sigma L .
$$

The first string derivation of Eq. (9) was proposed by Nambu [15]. The idea is as follows. Suppose that massless quarks rotate at the speed of light at radius $l / 2$. At the distance $r$ from the center of rotation the speed of flux tube connecting the quarks is $v(r)=2 r / l$. Then the mass of rotating gluon flux tube is

$$
M=2 \int_{0}^{l / 2} \frac{\sigma d r}{\sqrt{1-v^{2}(r)}}=\frac{\pi \sigma l}{2},
$$

while the angular momentum is

$$
L=2 \int_{0}^{l / 2} \frac{\sigma r v(r) d r}{\sqrt{1-v^{2}(r)}}=\frac{\pi \sigma l^{2}}{8} .
$$

Combining Eqs. (10) and (11) one arrives at Eq. (9).

The next step is to quantize the quasiclassical rotating string. This is a rather controversial problem in the literature. In particular, one should reconcile a different factor of proportionality between the mass $M$ and the length of gluon flux tube $l$ in Eqs. (44) and (10). Usually one obtains the following relation (at least for large $L$ and $n$ )

$$
M^{2}=2 \pi \sigma(L+b n+c),
$$

where the parameters $b$ and $c$ are model dependent. The factor $b$ lies typically in the interval $1 \leq b \leq 2$ [16-19]. At integer values of $b$, in particular $b=1$ and $b=2$, the specific spectral degeneracies emerge at different $(L, n)$. In the case $b=2$ the degeneracy is of the harmonic oscillator type. For $b=1$ one obtains the Coulomb-like degeneracy, where the energy levels (masses) depend only on the "principal quantum number" $N$,

$$
N=L+n+1 .
$$


Let us discuss briefly the intercept $2 \pi \sigma c$. For spinless constituents the physical meaning of this quantity seems to be the same as in the nonrelativistic Quantum Mechanics: It is the energy of zero-point oscillations of quarks inside a meson, which appear due to the uncertainty relation. In the reality, however, quarks do possess the spin. The resulting spin-spin interaction could give a substantial contribution to the mass of the ground S-wave mesons, where the quark/antiquark spin wave function have a maximal region of overlap. Presently, there is no complete understanding of underlying dynamics.

Finally, some comments are in order. In considering a spectrum like in Eq. (1) (with Eq. (2)) the following objection appears immediately: In relativistic systems, the internal angular momentum and spin cannot be separated, so why we should trust in this model? However, there are physical arguments that the spin-orbital correlations are asymptotically suppressed in excited hadrons [5, 6, 20-23]. In this case the usual quantum-mechanical rules for classifying the composite states seem to be applicable. In fact, it has been known for long ago that the nonrelativistic classification of light mesons works rather well $[24,25]$. What is important for us is that this classification predicts the doubling of states in the channels where the resonances can be created by different angular momentum. For instance, the vector mesons can have $L=0$ and $L=2$ (the so-called S- and D-wave mesons in the nonrelativistic spectroscopy), hence, they are doubled. Experimentally such a doubling is well seen. In practice one achieves the separation of resonances into the states with different angular momentum by using the polarization data. In particular, a good separation was obtained for the states with $(C, I)=(+1,0),(-1,1)$ in the Crystal Barrel experiment [26,27]. For other channels with duplication of states the experimental separation is not so clear. We note also that the spectrum under consideration can be cast into a relativistic form due to Eq. (2),

$$
M^{2}(J, n) \sim J+n+c .
$$

In this case, however, the constant $c$ is not approximately universal for all channels.

Another objection comes from the lattice calculations which typically point out a string breaking in the static potential between two quarks at distances of about $1-1.5 \mathrm{fm}$. In the potential models one usually mimics the phenomenon by a screened linear potential (see, e.g., [28]). In this respect we remind that the QCD forces can be modeled by the static potential only for heavy enough quarks. The extrapolation of these results to the light quark sector, generally speaking, is not justified since the nonstatic contri- 
butions, say, the velocity-dependent terms, are expected to be important for the ultrarelativistic quarks, if not decisive.

\section{Phenomenological analysis}

In this section we will estimate the constants in Eq. (12) from the phenomenology. The procedure would be quite straightforward if many reliable experimental data were available. The Particle Data [1] contains a number of reliable non-strange mesons below $1.9 \mathrm{GeV}$. Above that region the experimental situation is worse. The only experiment which systematically looked for the non-strange resonances above $1.9 \mathrm{GeV}$ (in the region 1.9-2.4 GeV) was the one carried out by the Crystal Barrel Collaboration on the protonantiproton annihilation in flight, the corresponding results are summarized in a review $[26,27]$. The Particle Data cites these results in the section "Further States".

As a basis we take the analysis performed in [4], where the spectroscopy of non-strange mesons from the Particle Data (below $1.9 \mathrm{GeV}$ ) and Crystal Barrel (in the range 1.9-2.4 GeV) was analysed. For completeness we add the one-star states from [26] and also the $\rho(1900)$ and $h_{1}(1595)$ mesons. We exclude the exotic $\pi_{1}$-states. Three such mesons are known with the masses $1376 \pm 17,1653_{-15}^{+18}$, and $2013 \pm 25 \mathrm{MeV}$. Although the masses of these states agree nicely with the cluster structure of meson mass distribution, a definite $(L, n)$-assignment cannot be done for them within the standard Quark Model. Finally, the states we use in the present analysis are given in Table 1.

Now we must provide a definite $(L, n)$-assignment for the states in Table 1 . For some mesons the value of angular momentum $L$ is dictated by the quantum numbers of the quark-antiquark pair. Say, the axial-vector mesons can have only $L=1$, etc. For other mesons two different values of $L$ can be assigned, for instance, the vector mesons can have $L=0$ and $L=2$. The problem appears, how they can be distinguished? This problem is indeed serious for the energy region above $1.7 \mathrm{GeV}$, where the doubling of states with identical quantum numbers in a close mass interval emerges. As discussed at the end of Sect. 2, for the states with $(C, I)=(+1,0),(-1,1)$ the angular momentum assignment is provided, in many cases, by the polarisation data [26]. For other states we use a general principle: The states with a larger $L$ have a larger centrifugal barrier, which pushes up the corresponding masses. Finally, our assignment in $(L, n)$ is displayed in Table 2. Reading the data in Table 2 in a diagonal way (for the sake of convenience we introduced different frames) one can immediately notice an approximate degeneracy of states with the same $L+n$, just as predicted by Eq. (1) if $b=1$. The data 
Table 1: The masses (in MeV) of states from [1] and [26]. Experimental errors are indicated. Each column corresponds to a cluster in Fig. 1.

\begin{tabular}{|c|c|c|c|c|c|}
\hline $\begin{array}{l}\pi \\
\eta\end{array}$ & 135 & $\begin{array}{l}1300 \pm 100 \\
1294 \pm 4\end{array}$ & $\begin{array}{l}1812 \pm 14 \\
1760 \pm 11\end{array}$ & $\begin{array}{l}2070 \pm 35 \\
2010_{-60}^{+35}\end{array}$ & $\begin{array}{l}2360 \pm 25 \\
2285 \pm 20\end{array}$ \\
\hline$\omega$ & $782.65 \pm 0.12$ & $1400 \div 1450$ & $1670 \pm 30$ & $1960 \pm 25$ & $\begin{array}{l}2205 \pm 30 \\
2295 \pm 50\end{array}$ \\
\hline$\rho$ & $775.5 \pm 0.4$ & $1459 \pm 11$ & $1720 \pm 20$ & $\begin{array}{l}1900 \pm ? \\
2000 \pm 30\end{array}$ & $\begin{array}{l}2110 \pm 35 \\
2265 \pm 40\end{array}$ \\
\hline $\begin{array}{l}f_{0} \\
a_{0}\end{array}$ & & $\begin{array}{l}1200 \div 1500 \\
1474 \pm 19\end{array}$ & $1770 \pm 12$ & $\begin{array}{l}2020 \pm 38 \\
2025 \pm 30\end{array}$ & $2337 \pm 14$ \\
\hline$a_{1}$ & & $1230 \pm 40$ & $1647 \pm 22$ & $1930_{-70}^{+30}$ & $2270_{-40}^{+55}$ \\
\hline$f_{1}$ & & $1281.8 \pm 0.6$ & & $1971 \pm 15$ & $2310 \pm 60$ \\
\hline$h_{1}$ & & $1170 \pm 20$ & $1595 \pm 20$ & $1965 \pm 45$ & $2215 \pm 40$ \\
\hline$b_{1}$ & & $1229.5 \pm 3.2$ & $1620 \pm 15$ & $1960 \pm 35$ & $2240 \pm 35$ \\
\hline$f_{2}$ & & $1275.4 \pm 1.1$ & $1638 \pm 6$ & $\begin{array}{l}1934 \pm 20 \\
2001 \pm 10\end{array}$ & $\begin{array}{l}2240 \pm 15 \\
2293 \pm 13\end{array}$ \\
\hline$a_{2}$ & & $1318.3 \pm 0.6$ & $1732 \pm 16$ & $\begin{array}{l}1950 \pm 40 \\
2030 \pm 20\end{array}$ & $\begin{array}{l}2175 \pm 40 \\
2255 \pm 20\end{array}$ \\
\hline$\pi_{2}$ & & & $\begin{array}{l}1672.4 \pm 3.2 \\
1617+5\end{array}$ & $\begin{array}{l}2005 \pm 15 \\
2030+16\end{array}$ & $\begin{array}{l}2245 \pm 60 \\
2267+14\end{array}$ \\
\hline$\omega_{3}$ & & & $1667 \pm 4$ & $\begin{array}{l}2030 \pm 10 \\
1945 \pm 20\end{array}$ & $2255 \pm 15$ \\
\hline$\rho_{3}$ & & & $1688.8 \pm 2.1$ & $1982 \pm 14$ & $\begin{array}{l}2300_{-80}^{+50} \\
2260 \pm 20\end{array}$ \\
\hline$\omega_{2}$ & & & & $1975 \pm 20$ & $2195 \pm 30$ \\
\hline$\rho_{2}$ & & & & $1940 \pm 40$ & $2225 \pm 35$ \\
\hline$f_{3}$ & & & & $2048 \pm 8$ & $2303 \pm 15$ \\
\hline$a_{3}$ & & & & $2031 \pm 12$ & $2275 \pm 35$ \\
\hline$h_{3}$ & & & & $2025 \pm 20$ & $2275 \pm 25$ \\
\hline$b_{3}$ & & & & $2032 \pm 12$ & $2245 \pm 50$ \\
\hline$a_{4}$ & & & & $2005_{-45}^{+25}$ & $2255 \pm 40$ \\
\hline$f_{4}$ & & & & $2018 \pm 6$ & $2283 \pm 17$ \\
\hline$\omega_{4}$ & & & & & $2250 \pm 30$ \\
\hline$\rho_{4}$ & & & & & $2230 \pm 25$ \\
\hline$\pi_{4}$ & & & & & $2250 \pm 15$ \\
\hline$\eta_{4}$ & & & & & $2328 \pm 38$ \\
\hline$\omega_{5}$ & & & & & $2250 \pm 70$ \\
\hline$\rho_{5}$ & & & & & $2300 \pm 45$ \\
\hline
\end{tabular}


is visualized in Fig. 1, with the experimental errors being indicated. The clusters of states formed at fixed $L+n$ are distinctively seen.

Let us estimate quantitatively to what extent the outlined picture works. For this purpose we perform the following procedure. First, we take the quadratic mean values for the masses in each cell of Table 2 . The result is given in Table 3. Second, assuming the parametrization of spectrum dictated by Eq. (12),

$$
M^{2}(L, n)=A L+B n+C,
$$

we find the best fit for the plane $M^{2}(L, n)$ by means of the least-squares method applied to two variables $(L, n)$, with the values being taken from Table 3. The parametrization (15) is not expected to be operative for the $(0,0)$-states, thus, they are excluded from the fit. Keeping in mind the discussions in the previous section, we distinct three cases: the whole spectrum $\left(\bar{M}^{2}\right)$, the spectrum for the S-wave states $\left(\bar{M}_{s}^{2}\right)$, and the one for the states with $L>0\left(\bar{M}_{n s}^{2}\right)$. The results of our fit are presented in Table 4.

Thus, our phenomenological analysis confirms the law

$$
M^{2}(L, n) \sim L+n
$$

for the spectrum of mesons composed of the up and down quarks. Of course, our result is obtained for a particular $(L, n)$-assignment of the states, which is not unique. Indeed, as can be seen from Table 2 and/or Fig. 1, the assignment of some S-wave states to a particular cluster is ambiguous, hence, they can be reshuffled in various ways in the cells of Table 2. However, since the statistical weight of these states is relatively small, this ambiguity does not change the result drastically. The exclusion of one-star states does not affect the fit strongly as well. In any case the value of parameter $b$ Eq. (1) lies in the interval

$$
0.9<b<1.1
$$

with a rather high confidence level, i.e., it is compatible with $b=1$ within the experimental errors.

\section{Discussions}

The spectrum (16) (more exactly, its relativistic form, Eq. (14)) was first obtained within the old dual amplitudes $[9,10,29]$. Later it was realized that this spectrum is a feature of the relativistic Nambu-Goto open string since the frequencies of classical oscillatory and rotational motions of this string coincide at the same energy (see, e.g., [30]). Recently the spectral law (16) was reported within the AdS/QCD approach [31-33], in the latter reference 
Table 2: Classification of light non-strange mesons according to the values of $(L, n)$. The states with the lowest star rating (according to [26]) or which are doubtful as non-strange quark-antiquark resonances are marked by the question mark.

\begin{tabular}{|c|c|c|c|c|c|}
\hline$L_{L}{ }^{n}$ & 0 & 1 & 2 & 3 & 4 \\
\hline 0 & $\begin{array}{l}\pi(140) \\
\rho(770) \\
\omega(780)\end{array}$ & $\begin{array}{c}\pi(1300) \\
\rho(1450)(?) \\
\omega(1420)(?) \\
\eta(1295)\end{array}$ & $\begin{array}{l}\pi(1800) \\
\eta(1760)\end{array}$ & $\begin{array}{c}\pi(2070) \\
\rho(1900)(?) \\
\eta(2010) \\
\end{array}$ & $\begin{array}{c}\pi(2360) \\
\rho(2150) \\
\omega(2205)(?) \\
\eta(2285)\end{array}$ \\
\hline 1 & $\begin{array}{c}f_{0}(1370) \\
a_{0}(1450)(?) \\
a_{1}(1260) \\
f_{1}(1285) \\
b_{1}(1230) \\
h_{1}(1170) \\
a_{2}(1320) \\
f_{2}(1275)\end{array}$ & $\begin{array}{c}f_{0}(1770) \\
a_{1}(1640) \\
b_{1}(1620)(?) \\
h_{1}(1595)(?) \\
a_{2}(1680) \\
f_{2}(1640)\end{array}$ & $\begin{array}{c}f_{0}(2020) \\
a_{0}(2025) \\
a_{1}(1930)(?) \\
f_{1}(1971) \\
b_{1}(1960) \\
h_{1}(1965) \\
a_{2}(1950)(?) \\
f_{2}(1934)\end{array}$ & $\begin{array}{c}f_{0}(2337) \\
a_{1}(2270)(?) \\
f_{1}(2310) \\
b_{1}(2240) \\
h_{1}(2215) \\
a_{2}(2175)(?) \\
f_{2}(2240)\end{array}$ & \\
\hline 2 & $\begin{array}{l}\rho(1700) \\
\omega(1650) \\
\pi_{2}(1670) \\
\eta_{2}(1645) \\
\rho_{3}(1690) \\
\omega_{3}(1670)\end{array}$ & \begin{tabular}{|c||}
$\rho(2000)$ \\
$\omega(1960)$ \\
$\pi_{2}(2005)$ \\
$\eta_{2}(2030)$ \\
$\rho_{2}(1940)$ \\
$\omega_{2}(1975)$ \\
$\rho_{3}(1982)$ \\
$\omega_{3}(1945)$ \\
\end{tabular} & $\begin{array}{c}\rho(2265) \\
\omega(2295)(?) \\
\pi_{2}(2245) \\
\eta_{2}(2267) \\
\rho_{2}(2225) \\
\omega_{2}(2195) \\
\rho_{3}(2300)(?) \\
\omega_{3}(2285)\end{array}$ & & \\
\hline 3 & \begin{tabular}{l|}
$f_{2}(2001)$ \\
$a_{2}(2030)$ \\
$f_{3}(2048)$ \\
$a_{3}(2031)$ \\
$b_{3}(2032)$ \\
$h_{3}(2025)$ \\
$f_{4}(2018)$ \\
$a_{4}(2005)$
\end{tabular} & $\begin{array}{l}f_{2}(2293) \\
a_{2}(2255) \\
f_{3}(2303) \\
a_{3}(2275) \\
b_{3}(2245) \\
h_{3}(2275) \\
f_{4}(2283) \\
a_{4}(2255)\end{array}$ & & & \\
\hline 4 & $\begin{array}{c}\rho_{3}(2260) \\
\omega_{3}(2255) \\
\rho_{4}(2230) \\
\omega_{4}(2250)(?) \\
\pi_{4}(2250) \\
\eta_{4}(2328) \\
\rho_{5}(2300) \\
\omega_{5}(2250)\end{array}$ & & & & \\
\hline
\end{tabular}




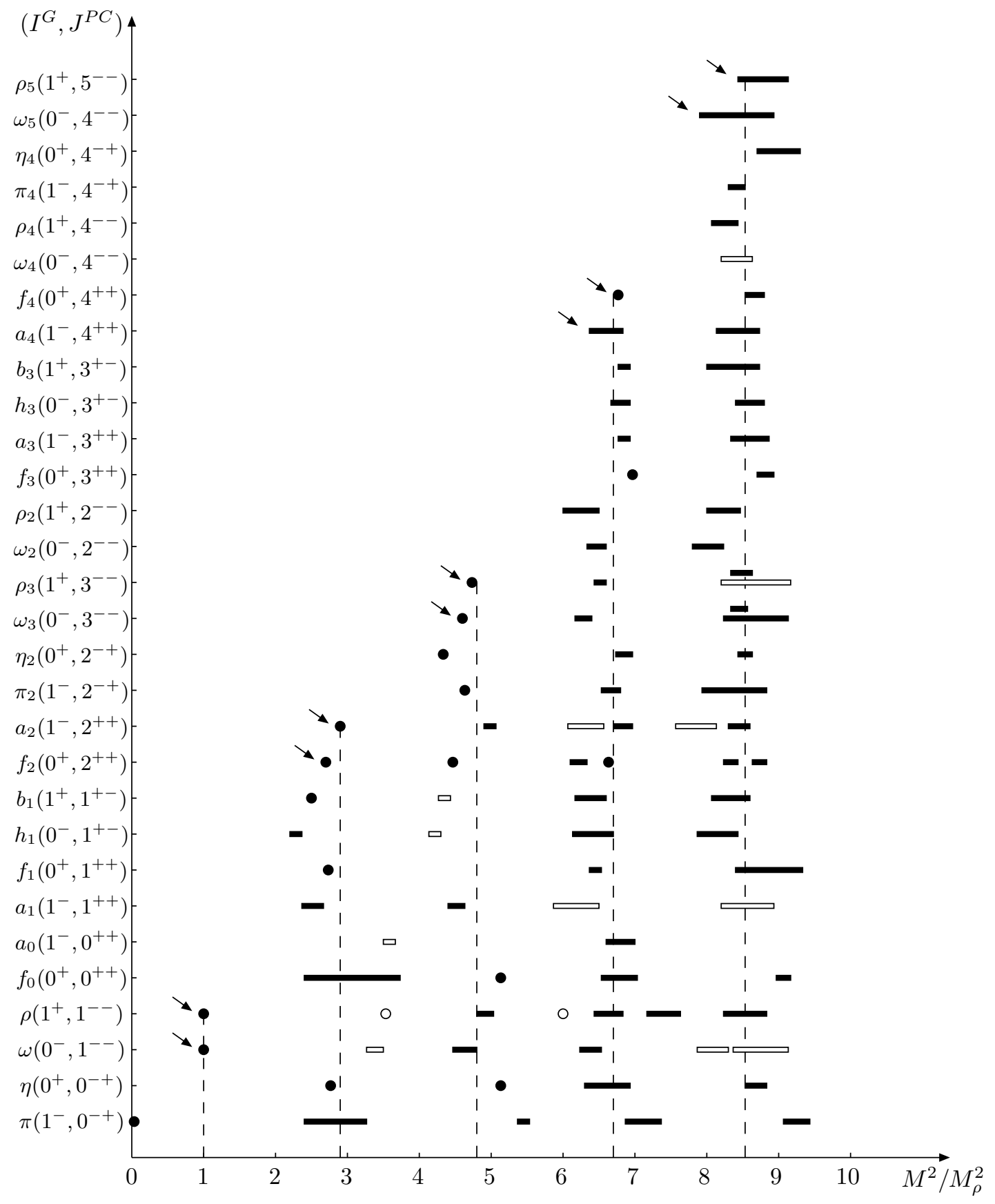

Figure 1: The spectrum of light non-strange mesons in units of $M_{\rho(770)}^{2}$. The data is taken from Table 1. Experimental errors are indicated. Circles stay when errors are negligible. The dashed lines mark the mean (mass) ${ }^{2}$ in each cluster of states and the open strips and circles denote the states marked as (?) in Table 2. The arrows indicate the $J>0$ mesons which have no chiral partners (the hypothetical chiral singlets, see the discussions in Sect. 4). 
Table 3: Quadratic mean values in the $(L, n)$ blocks of Table 2 (in MeV).

\begin{tabular}{|c|c|c|c|c|c|}
\hline$L^{n}$ & 0 & 1 & 2 & 3 & 4 \\
\hline 0 & - & 1373 & 1780 & 1995 & 2242 \\
\hline 1 & 1294 & 1668 & 1970 & 2256 & \\
\hline 2 & 1673 & 1980 & 2260 & & \\
\hline 3 & 2024 & 2273 & & & \\
\hline 4 & 2266 & & & & \\
\hline
\end{tabular}

Table 4: The spectrum obtained by the least-squares method (in $\mathrm{GeV}^{2}$ ). The last column displays the predicted mass of the ground state (in $\mathrm{GeV}$ ).

\begin{tabular}{l|c|c|c}
\hline \hline & $A L+B n+C$ & $a(L+b n+c)$ & $\sqrt{C}$ \\
\hline $\bar{M}^{2}$ & $1.103 L+1.102 n+0.686$ & $1.103(L+n+0.622)$ & 0.828 \\
$\bar{M}_{n s}^{2}$ & $1.178 L+1.135 n+0.473$ & $1.178(L+0.963 n+0.402)$ & 0.688 \\
$\bar{M}_{s}^{2}$ & $1.023 n+0.957$ & $1.023(n+0.935)$ & 0.978 \\
\hline \hline
\end{tabular}

a holographic dual of QCD was proposed which gives the slope $\frac{1}{2}$ just as the WKB method. The existing quark models are unable to reproduce Eq. (16), as was emphasized recently in [34], a solution of this problem is a challenge for future quark models. In a broad sense, spectrum of any such model should depend on a single "principal quantum number" (13). An attempt to relate the existence of this quantum number to confinement realized through the area law for Wilson loops was undertaken in [35].

In the last column of Table 4 we give an extrapolation of obtained mass spectra to the mass of $(0,0)$-mesons for $L=0$ and $L>0$ states. The experimental masses of $\rho(770)$ and $\omega(782)$ mesons lie in between. This could mean that the physical vector ground states represent a strong mixture of both S-wave and D-wave components. On the other hand, it may be that the deviation from the prediction is caused mainly by the quark/antiquark spin-spin interaction. Within the WKB method a mass of the ground state is directly related to a value of constant $\gamma$ in Eq. (5). In this respect it is interesting to note that the values of parameter $c$ in Table 4 are close to those of $\gamma$ given by the WKB approximation without account of both the quark spin and the string rotation. Thus, we see that a simple application of semiclassical methods yields the result which is rather close to the averaged experimental one. It would be interesting to develop the subject in future. 
Given the phenomenological fit, a question appears: Which effective string model proposed in the literature is in the best agreement with the experiment? We think that this is a model advocated in [19]. First of all, it reproduces Eq. (14). Second, for the mesons with $L=J+1$ this model (if one leaves the quantization condition $J=L+\frac{1}{2}$ exploited in [19]) gives an intercept close to the reality: $c=\frac{5}{12} \approx 0.417$.

The next issue we consider is the asymptotic chiral symmetry restoration in the highly excited states. Namely, we would like to draw attention to the following aspect of the problem: All states on the lowest leading Regge trajectories (the $\rho, \omega, f_{2}$, and $a_{2}$ trajectories) do not possess the chiral partners. In Fig. 1 these states reside on the top of the depicted meson clusters and they are indicated by arrows. One can make even a stronger statement: In the case of states belonging to these trajectories there are no particles with equal spin in a close mass region. This experimental fact (yet not well established) contradicts to the usual effective chiral symmetry restoration scenario (for a recent review see [6]) where each light hadron has to have a chiral partner with close mass at high enough energies, approximately since $1.7 \mathrm{GeV}$ (we remind that the chiral transformations relate particles with equal spins). Meanwhile, the phenomenon is a natural consequence of string-like nonrelativistic spectral formulas like Eq. (16): Since the parity of quark-antiquark system is defined as

$$
P=(-1)^{L+1},
$$

one of parity-conjugated meson towers has a lower $L$, hence, the ground state in this tower is sentenced to be a parity bachelor. This mechanism naturally implements the chiral symmetry breaking in the hadron spectrum. For instance, the ground $\rho$ - and $a_{1}$-mesons cannot be degenerate because they are $(0,0)$ and $(1,0)$ states correspondingly (in the notation $(L, n))$ and the sum $L+n$ is different. This effect is repeated at larger $L$ for the states on the leading trajectories, up to the highest available mass range.

Thus, the absence of chiral partners for the leading meson trajectories seems to be an experimental fact, nevertheless, is this a law of Nature? A possible answer is negative - just nobody in the past performed a systematic search for these partners. Say, some states on the leading nucleon Regge trajectory possess the parity doublers with a close mass. However, at present the absence of parity doubling on the leading meson Regge trajectories is a rather definite experimental result 1 . If the chiral symmetry gets effectively restored since approximately $1.7 \mathrm{GeV}$, then the states $\rho_{3}(1690)$ and $\omega_{3}(1670), f_{4}(2050)$ and $a_{4}(2005), \rho_{5}(2300)$ and $\omega_{5}(2250)$ have to possess the chiral partners. The resonances $\rho_{3}(1690)$ and $\omega_{3}(1670)$ are well known, but nobody observed the

\footnotetext{
${ }^{1}$ I am grateful to D. V. Bugg for elucidating me this point.
} 
corresponding parity partners, which ought to be very easy to find. Instead, the Crystal Barrel revealed very conspicuous $a_{3}(2031)$ and $f_{3}(2048)$, which are the parity partners of $\rho_{3}(1982)$ and $\omega_{3}(1945)$ correspondingly. There is no sign of anything near the well seen resonances $f_{4}(2050)$ and $\rho_{5}(2300)$ in the Crystal Barrel. Thus, the only experiment designed for the systematic search of the non-strange meson resonances above $1.9 \mathrm{GeV}$ did not discover the chiral partners for the states on the leading trajectories. One should wait for another similar experiment to confirm or refute the result.

As discussed above, the hadron string picture naturally leads to the existence of chiral-singlet states if one accepts the standard definition of parity for the quark-antiquark system, Eq. (18). A possible objection is that this definition is nonrelativistic. It may be that if one redefines the parity somehow then the chiral-singlet states will disappear from the hadron string spectrum and the latter will be compatible with a usual chiral symmetry restoration scenario (for a recent attempt see [23]). Our conclusion concerning the experimental existence of chiral singlets, however, does not depend on a concrete definition of parity: if there is no state with equal spin in a close vicinity of some hadron, then this hadron is a chiral singlet. But what does "close" mean? The cluster structure of mass distribution seen in Fig. 1 provides an answer to this question: There is no state with equal spin in the given cluster, i.e., the notion "close" is defined with respect to the typical distance between two neighbouring clusters.

Finally, it should be emphasized that the existence of chiral singlets does not necessary contradict to the effective chiral symmetry restoration in the highly excited states. Say, the symmetry particle-antiparticle has singlets, e.g., the $\pi^{0}$-meson. The situation with the chiral symmetry could be somewhat similar.

\section{Conclusions}

We have reviewed briefly some recent attempts to understand a broad degeneracy existing in the spectra of light non-strange mesons, the X-symmetry. The degeneracy can be summarized compactly as the spectral law $M^{2}(L, n) \sim$ $L+n$, which we checked using the latest experimental information and the agreement is found to be remarkable. Needless to say that this law, if true, is of great importance - it explains the X-symmetry and plays a powerful selective role both for the model building and for the hadron systematics. For instance, it seems to be not compatible with a recently proposed $\tilde{U}(12)_{S F} \times S O(3,1)_{L}$ classification scheme for mesons [36], which predicts the spectrum (see also [37]) $M^{2}(L, n) \sim L+2 n$. 
A natural candidate for a dynamical model reproducing the expected mass spectrum and, hence, generating the X-symmetry is argued to be an effective hadron string. At present it is not clear if the hadron strings really exist as physical objects. It may be that looking at hadrons through the magnifying glass we would see something different. Nevertheless, the strings are shown to emerge quite naturally as an organizing principle for hadron spectroscopy. The concept of hadron strings combines the theoretical simplicity and explicative power, in this respect it seems to be unrivalled presently, although some interesting alternative approaches exist as well.

We demonstrated that a constant correction (the intercept) to the spectrum under consideration, Eq. (16), can be understood quasiclassically, namely, within the WKB approximation. This supports the idea that the highly excited states are, in essence, semiclassical systems.

The hypothetical X-symmetry seems to include the asymptotic chiral and axial symmetries as a particular case. On the other hand, given the modern experimental data, the X-symmetry should lead to the existence of chiral singlet states. A development of this subject is a challenge for future models aiming at the description of meson spectra.

\section{Acknowledgments}

I am grateful to M. Shifman and A. Vainshtein for private communications and to the Department of Structure and Constituents of the Matter (especially to Prof. D. Espriu) of Barcelona University, where a part of the work was carried out. The work was supported by CYT FPA, grant 2004-04582C02-01, CIRIT GC, grant 2001SGR-00065, RFBR, grant 05-02-17477, grant LSS-5538.2006.2, and by the Ministry of Education of Russian Federation, grant RNP.2.1.1.1112.

\section{References}

[1] Particle Date Group (W.-M. Yao et al.), J. Phys. G 33, 1 (2006).

[2] S. S. Afonin, Phys. Lett. B 639, 258 (2006) hep-ph/0603166.

[3] S. S. Afonin, arXiv:0704.1639 [hep-ph].

[4] S. S. Afonin, Eur. Phys. J. A 29, 327 (2006) [hep-ph/0606310].

[5] M. Shifman and A. Vainshtein, in preparation. 
[6] L. Ya. Glozman, hep-ph/0701081.

[7] L. D. Landau and E. M. Lifshitz, Course of Theoretical Physics (Pergamon Press, Oxford, 1975) Vol. 3.

[8] V. L. Morgunov, A. V. Nefediev, and Yu. A. Simonov, Phys. Lett. B 459, 653 (1999).

[9] C. Lovelace, Phys. Lett. B 28, 264 (1968).

[10] J. A. Shapiro, Phys. Rev. 179, 1345 (1969).

[11] S. S. Afonin, Phys. Lett. B 576, 122 (2003) [hep-ph/0309337.

[12] S. S. Afonin, to be published in Nucl. Phys. B [hep-ph/0606291].

[13] S. S. Afonin and D. Espriu, JHEP 09, 047 (2006) hep-ph/0602219].

[14] S. S. Afonin, A. A. Andrianov, V. A. Andrianov, and D. Espriu, JHEP 04, 039 (2004) [hep-ph/0403268].

[15] Y. Nambu, Phys. Rev. D 210, 4262 (1974).

[16] J. S. Kang and H. J. Schnitzer, Phys. Rev. D 12, 841 (1975).

[17] C. Goebel, D. LaCourse, and M. G. Olsson, Phys. Rev. D 41, 2917 (1990).

[18] F. Brau, Phys. Rev. D 62, 014005 (2000).

[19] M. Baker and R. Steinke, Phys. Rev. D 65, 094042 (2002).

[20] A. Selem and F. Wilczek, hep-ph/0602128.

[21] F. Wilczek, hep-ph/0409168.

[22] N. Matagne and F. Stancu, Phys. Rev. D 71, 014010 (2005).

[23] L. Ya. Glozman, Phys. Lett. B 541, 115 (2002).

[24] S. Godfrey and J. Napolitano, Rev. Mod. Phys. 71, 1411 (1999).

[25] A. V. Anisovich, V. V. Anisovich, and A. V. Sarantsev, Phys. Rev. D 62, 051502(R) (2000).

[26] D. V. Bugg, Phys. Rept. 397, 257 (2004).

[27] V. V. Anisovich, Phys. Usp. 47, 45 (2004) hep-ph/0208123. 
[28] P. González, J Vijande, A. Valcarce, and H. Garcilazo, Eur. Phys. J. A 29, 235 (2006).

[29] G. Veneziano, Nuovo Cim. A 57, 190 (1968).

[30] B. Zwiebach, A First Course in String Theory (Cambridge University Press, Cambridge, 2004).

[31] A. Karch, E. Katz, D. T. Son, and M. A. Stephanov, Phys. Rev. D 74, 015005 (2006).

[32] H. Forkel, M. Beyer, and T. Frederico, arXiv:0705.1857 [hep-ph].

[33] H. Forkel, M. Beyer, and T. Frederico, arXiv:0705.4115 [hep-ph].

[34] P. Bicudo, hep-ph/0703114.

[35] Yu. A. Simonov, Phys. Lett. B 226, 151 (1989).

[36] S. Ishida, M. Ishida, and T. Maeda, Prog. Theor. Phys. 104, 785 (2000).

[37] K. Yamada, hep-ph/0612337. 